\title{
KRAS mutations in patients with colorectal cancer in Libya
}

\author{
ASMA ABUDABOUS ${ }^{1}$, MUSTAFA DRAH $^{2}$, MAMDOUH ALDEHMANI $^{3}$, IQBAL PARKER $^{4}$ and OMAR ALQAWI ${ }^{5}$ \\ ${ }^{1}$ Department of Life Sciences, The Libyan Academy; ${ }^{2}$ Department of Zoology, Faculty of Science, Misurata University; \\ ${ }^{3}$ National Cancer Institute-Misurata, Misurata 218-51, Libya; ${ }^{4}$ Department of Integrative Biomedical Sciences and \\ Institute of Infectious Disease and Molecular Medicine, Faculty of Health Sciences, University of Cape Town, \\ Cape Town 7925, South Africa; ${ }^{5}$ Biotechnology Research Centre, National Cancer Institute-Misurata, Misurata 218-51, Libya
}

Received August 19, 2020; Accepted December 17, 2020

DOI: $10.3892 / \mathrm{mco} .2021 .2359$

\begin{abstract}
Large prospective clinical trials have demonstrated that colorectal cancers (CRCs) with wild-type KRAS respond favorably to anti-epidermal growth factor receptor treatment, thus making mutational analysis obligatory prior to treatment. In our study, frozen CRC tissues from Libyan patients were analyzed for KRAS and HRAS mutations in codons 12/13 by direct sequencing and the correlations with clinical and pathological parameters were investigated. A total of 34 CRC cases, comprising 19 men and 15 women (age range, 24-87 years), were subjected to systematic analysis for RAS mutations. Although HRAS mutations were not detected in any of the patients in the study group, KRAS codon 12/13 mutations were present in $38.2 \%$ (13/34) of the patients. The frequent types of codon 12 mutations were glycine to aspartate (G12D, 46.1\%); glycine to valine (G12V, 30.8\%) and glycine to cysteine (G12C, 15.4\%), while the codon 13 mutations were glycine to aspartate (G13D, 7.7\%). $\mathrm{G} \rightarrow \mathrm{A}$ mutations occurred in $53.8 \%(7 / 13)$ of the patients, while $\mathrm{G} \rightarrow \mathrm{T}$ mutations occurred in $46.2 \%(6 / 13)$ of the patients. Mutations occurred at the first base of codon 12 or 13 in 2/13 (15.4\%) and at the second base in 11/13 (84.6\%) patients. There was no significant association between clinicopathological characteristics and KRAS mutation status, except the site of the tumors harboring KRAS mutations, which was as follows: The frequency was higher among tumors located in the left colon $(8 / 13,61.5 \%)$ compared to other sites $(\mathrm{P}=0.027)$. KRAS mutations were correlated with advanced age, with $10 / 13$ being aged $>50$ years and affected $8 / 15$ female patients (53\%) compared with $5 / 19$ male patients (26\%). The highest frequency of KRAS mutations was observed in highly differentiated CRCs (8/13).
\end{abstract}

Correspondence to: Dr Omar Alqawi, Biotechnology Research Centre, National Cancer Institute-Misurata, 11 Nosoor Aljaw Street, Algheran, Misurata 218-51, Libya

E-mail: oalqawi@hotmail.com

Key words: colorectal cancer, KRAS, HRAS, mutation, DNA sequencing

\section{Introduction}

Colorectal cancer (CRC) is the 3rd and 4th most commonly diagnosed cancer globally in women and men, respectively (1-3). The transformation of the normal colonic epithelium is the result of the progressive accumulation of genetic and epigenetic alterations that promote tumor growth. Colon cancer cells result from a multi-step progression in molecular and morphological changes of normal cells. In addition, genetic instability and germ line genetic defects drive the initiation of sporadic colon cancers (4-6).

The RAS oncogene family, comprising three known human isoforms, NRAS, HRAS and KRAS, regulates a number of cellular functions, including cell proliferation, apoptosis, migration and differentiation (7). KRAS mutations comprise $86 \%$ of all RAS mutations in human cancers (8). The frequency of KRAS mutations is the highest (21.6\%) in all cancers, followed by NRAS (8.0\%) and HRAS (3.3\%) (9). The hotspot of KRAS mutations is located in exon 2 , and the most frequent changes are identified in codons 12 ( $82 \%$ of all reported KRAS mutations) and $13(\sim 17 \%)$. Other mutations of KRAS have been detected in codons 61 and 146, but these have little or no effect on the progression of CRC (10). The most frequent change is the transition of GGT to GAT in codon 12, specifically DNA nucleotide mutations involving $\mathrm{G} \rightarrow \mathrm{A}$ or $\mathrm{G} \rightarrow \mathrm{T}$ (7). However, $60 \%$ of NRAS mutations occur mainly in codon 61 vs. $35 \%$ in codon 12 , while HRAS mutations in CRC show an intermediate pattern with an approximate 50:40 split between mutations in codons 12 and 61, respectively (11). KRAS and HRAS mutations comprise single-nucleotide point mutations, with the most common substitutions in KRAS resulting in G12D, G12A, G12R, G12C, G12S, G12V and G13D substitutions and the most frequent codon 12 substitutions being G12D and G12V (10). However, in HRAS, the most common mutation results in a G12V substitution (11). The presence of a glycine residue in codon 12, therefore, plays an important role in the normal function of the RAS proteins. This single-nucleotide substitution that results in the replacement of glycine with another amino acid leads to the formation of a constitutively active GTPase (12).

RAS proteins are activated when nearby transmembrane receptors, such as growth factor receptors, G-protein coupled receptors and toll-like receptors, are bound by the corresponding ligand. The subsequent intracellular signaling cascade involves guanine exchange factors (GEF) that facilitate the activation of RAS by replacing the inactive GDP with GTP. RAS activation 
triggers the downstream activation of a wide variety of effectors, including serine/threonine kinases, GTPase-activating proteins (GAPs), phosphoinositide 3-kinase and GEFs. RAS is deactivated when the GTP is hydrolyzed to GDP. When GTP binds to the KRAS protein, KRAS undergoes a conformational change involving two regions of the protein known as Switch 1 (amino acids 30-38) and Switch 2 (amino acids 59-67), which form an effector loop controlling the specificity of the binding of this GTPase to its effector molecules. The changes in KRAS conformation affect its interactions with multiple downstream transducers, such as GAPs, which amplify the GTPase activity of RAS 100,000-fold (13). Once KRAS is mutated, it becomes constitutively active and the regulation of downstream functions is lost, resulting in unregulated cell growth (7). Screening of KRAS mutations is widely used in clinical practice to decide on the treatment for CRC (14). Recently, KRAS mutation screening has become common practice before prescribing anti-epidermal growth factor receptor monoclonal antibodies in the treatment of advanced CRC (15).

$\mathrm{CRC}$ is the second most common type of cancer among patients admitted to the National Cancer Institute(NCI)-Misurata, where $\sim 180$ new cases (52\% male and $48 \%$ female patients) were admitted in 2019. The incidence and mortality rates for CRC increase with age, with $>90 \%$ of the patients aged $>50$ years (2). The aim of the present study was to investigate the presence of KRAS and HRAS mutations among patients with CRC in NCI-Misurata, in order to determine whether the frequency of those mutations is associated with the type and stage of CRC.

\section{Materials and methods}

Tissue samples. The present study included 34 frozen tumor tissue samples obtained from patients with $\mathrm{CRC}$, who underwent curative surgery at NCI-Misurata. The patients were diagnosed at the Department of Histopathology between December 2016 and August 2017. The study protocol was approved by the Ethics Committee of NCI-Misurata. All tumor tissues were placed on ice immediately upon removal from the patient, sectioned and stored at $-80^{\circ} \mathrm{C}$ until DNA was extracted.

Clinical records. The clinical and demographic data collected from patient records included: Age, sex, family history of $\mathrm{CRC}$, tumor site, degree of tumor differentiation, tumor stage and histological type, such as common adenocarcinoma or mucinous carcinoma. Patients with family history of CRC were selected for the present study. All the patients have signed a consent form prior to the use of the studied tissues, according to the regulations of the NCI-Misurata.

DNA extraction and PCR reaction. Genomic DNA extraction from frozen tumor tissues was carried out using QIAamp DSP DNA Fresh Tissue kit (Qiagen $\mathrm{GmbH}$ ). After the washing steps, the DNA was eluted in Tris/EDTA buffer and stored at $-20^{\circ} \mathrm{C}$. The DNA was quantitated using a NanoDrop spectrophotometer (Thermo Fisher Scientific, Inc.). After extraction of the genomic DNA from the samples, the KRAS and HRAS exons land 2 were amplified using a PCR SimpliAmp ${ }^{\text {TM }}$ Thermal Cycler (Thermo Fisher Scientific, Inc.) using the forward 3'-AAGGCCTGCTGAAAATGACTG-5' and reverse 3'-CAA AGAATGGTCCTGCACCAG-5' primers for KRAS-exon 2 and the forward 3'-GGGCCCTCCTTGGCAGGTGG-5' and reverse 3'-CACCTGGACGGCGGCGCCAG-5' primers for HRAS-exon 2. Each PCR reaction was performed in a volume of $20 \mu \mathrm{l}$ containing PCR buffer, $2.5 \mathrm{mM} \mathrm{MgCl}_{2}, 5$ units of Taq polymerase, $1 \mathrm{mM}$ of each dNTP, forward and reverse primers (10 pmol) and $100 \mathrm{ng}$ of genomic DNA. The PCR reaction ran with the following program: $95^{\circ} \mathrm{C}$ initial denaturation for activating the Fast Star Taq DNA Polymerase for 5 min; step 2: Denaturation at $95^{\circ} \mathrm{C}$ for $30 \mathrm{sec}$; step 3: Annealing of the primers to the template at $54^{\circ} \mathrm{C}$ for $\mathrm{HRAS}$ in exons 1 and $2,45^{\circ} \mathrm{C}$ for KRAS in exon 1 , and $43^{\circ} \mathrm{C}$ for exon 2 for $30 \mathrm{sec}$; and step 4: $3 \mathrm{~min}$ at $72^{\circ} \mathrm{C}$ for primer extension. Steps 2-4 were repeated 25 times, followed by a final extension step at $72^{\circ} \mathrm{C}$ for $5 \mathrm{~min}$; subsequently, the PCR reaction was cooled down to $8^{\circ} \mathrm{C}$. The PCR products were subjected to direct DNA sequencing.

The amplification product sizes were confirmed by electrophoresis on $1 \%$ agarose gel in Tris-borate-EDTA buffer (Sigma-Aldrich; Merck KGaA), and visualized on a UVP BioSpectrum $^{\mathrm{TM}} 500$ Imaging System (VWR Corporation). The PCR reaction products were purified using the MinElute PCR Purification Kit (Qiagen $\mathrm{GmbH}$ ) and subjected to sequence analysis.

Direct DNA sequencing. Direct sequencing of PCR products was performed after purification with the PCR Product Purification Kit (Roche Diagnostics $\mathrm{GmbH}$ ). The forward primers used in PCR amplification of KRAS and HRAS genes were used for sequencing in on the EVO150 Genetic Analyzer (Tecan Group, Ltd./Applied Biosystems; Thermo Fisher Scientific, Inc.). To ensure the accuracy of sequencing results, all the molecular tests and the direct sequencing analysis were performed twice on each sample. All DNA sequencing reactions were performed by Inqaba Biotec.

Statistical analysis. Data analysis was performed using SPSS version 17.0 (SPSS Inc.). The Fisher's exact test was used for comparisons. $\mathrm{P}<0.05$ was considered to indicate a statistically significant difference.

\section{Results}

KRAS and HRAS mutations in histopathological biopsies. KRAS mutations were identified in 13/34 (38.2\%) of the tumors, while all the patients in this study group had the wild-type HRAS gene (Fig. 1). The site of the tumor was found to be associated with KRAS mutations $(\mathrm{P}=0.027)$. Tumors located in the left colon had a significantly higher frequency of mutant KRAS status $(8 / 11 ; 72.7 \%)$ compared with those located in the right colon. Table I shows the frequency and association with clinicopathological characteristics of patients who harbored mutant and wild-type KRAS tumors; $76.9 \%$ (10/13) of the patients with tumors harboring mutant KRAS presented with stage B, C or D colon cancer, indicating that patients with mutant KRAS tumors are more likely to have advanced-stage disease. Tumor differentiation status, sex, patient age and stage at diagnosis were not identified as significant predictors of KRAS status $(\mathrm{P}=0.458)$.

Type and frequency of KRAS gene mutations. The type and frequency of KRAS gene mutations are detailed in Table II. 
Table I. Distribution of tumour characteristics according to KRAS status.

\begin{tabular}{|c|c|c|c|c|}
\hline \multirow[b]{2}{*}{ Characteristics } & \multicolumn{3}{|c|}{ KRAS mutation status } & \multirow[b]{2}{*}{ P-value } \\
\hline & Overall & Mutant & Wild-type & \\
\hline Number of patients & 34 & 13 & 21 & \\
\hline Sex & & & & 0.107 \\
\hline Male & 19 & 5 & 14 & \\
\hline Female & 15 & 8 & 7 & \\
\hline Age (years) & & & & 0.524 \\
\hline$\leq 50$ & 10 & 3 & 7 & \\
\hline$>50$ & 24 & 10 & 14 & \\
\hline Localization & & & & 0.027 \\
\hline Right colon & 11 & 3 & 8 & \\
\hline Left colon & 11 & 8 & 3 & \\
\hline Rectosigmoid & 4 & 0 & 4 & \\
\hline Rectum & 8 & 2 & 6 & \\
\hline Differentiation & & & & 0.458 \\
\hline High & 16 & 8 & 8 & \\
\hline Moderate & 12 & 4 & 8 & \\
\hline Low & 2 & 0 & 2 & \\
\hline Unknown & 4 & 1 & 3 & \\
\hline Duke's stage & & & & 0.561 \\
\hline A & 3 & 2 & 1 & \\
\hline $\mathrm{B}$ & 7 & 4 & 3 & \\
\hline $\mathrm{C}$ & 18 & 5 & 13 & \\
\hline $\mathrm{D}$ & 3 & 1 & 2 & \\
\hline Unknown & 3 & 1 & 2 & \\
\hline
\end{tabular}

Table II. Distribution of KRAS mutation types.

\begin{tabular}{lcccc}
\hline Codon & Amino acid substitution & Incidence, $\%$ & Number of samples & Base change \\
\hline 12 & Gly12Asp & 46.1 & 6 & GGT $>$ GAT \\
12 & Gly12Val & 30.8 & 4 & GGT $>$ GTT \\
12 & Gly12Cys & 15.4 & 2 & GGT $>$ TGT \\
13 & Gly13Asp & 7.7 & 1 & GGC $>$ GAC \\
\hline
\end{tabular}

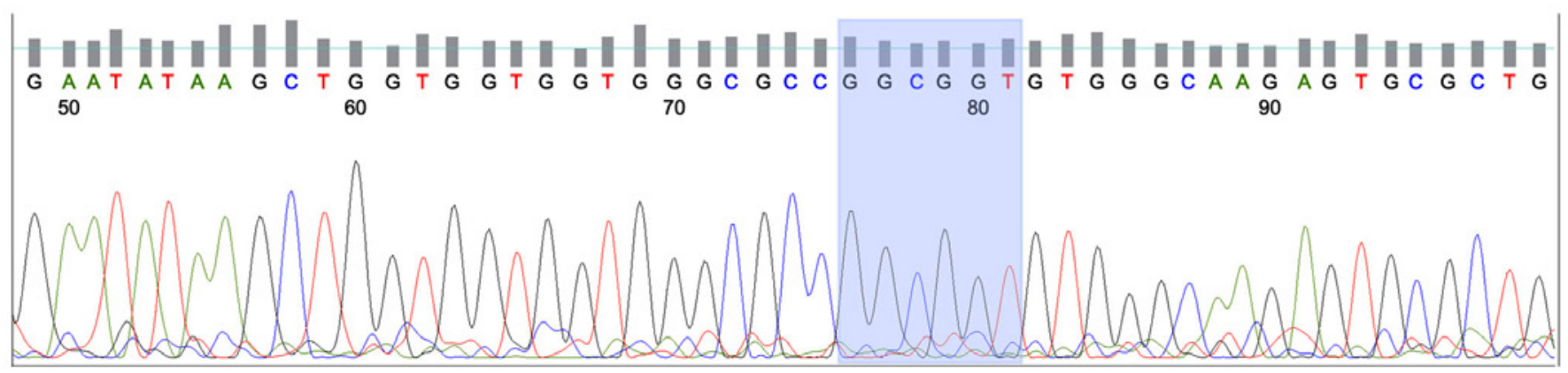

Figure 1. Sanger sequencing electropherogram showing the wild-type HRAS codons 12 and 13 (GGCGGT). No HRAS mutations were detected in any of the patients in the cohort. 
A

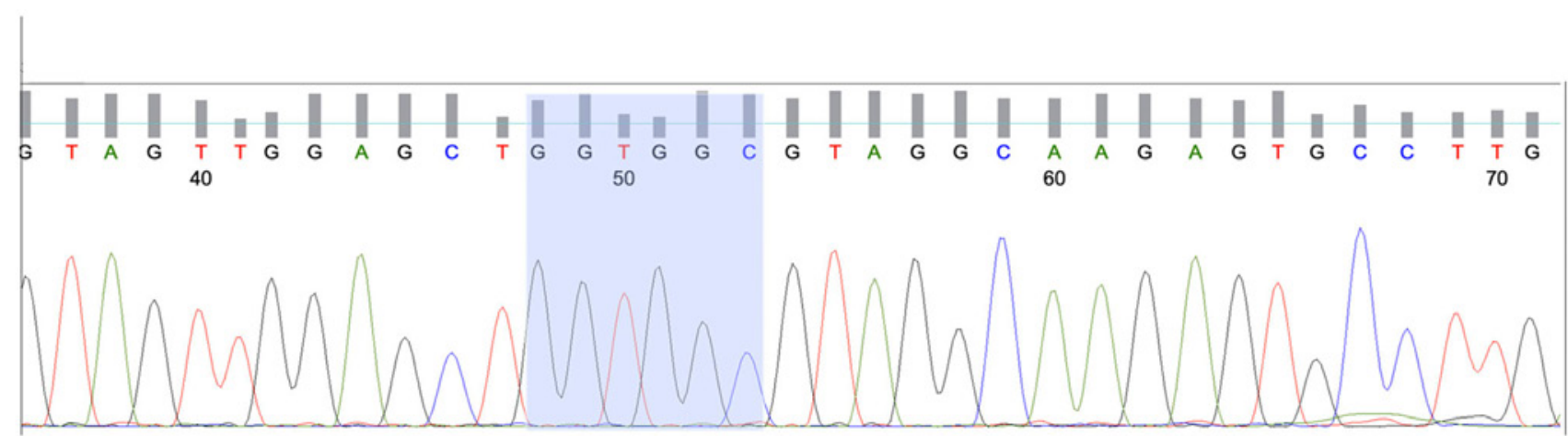

B

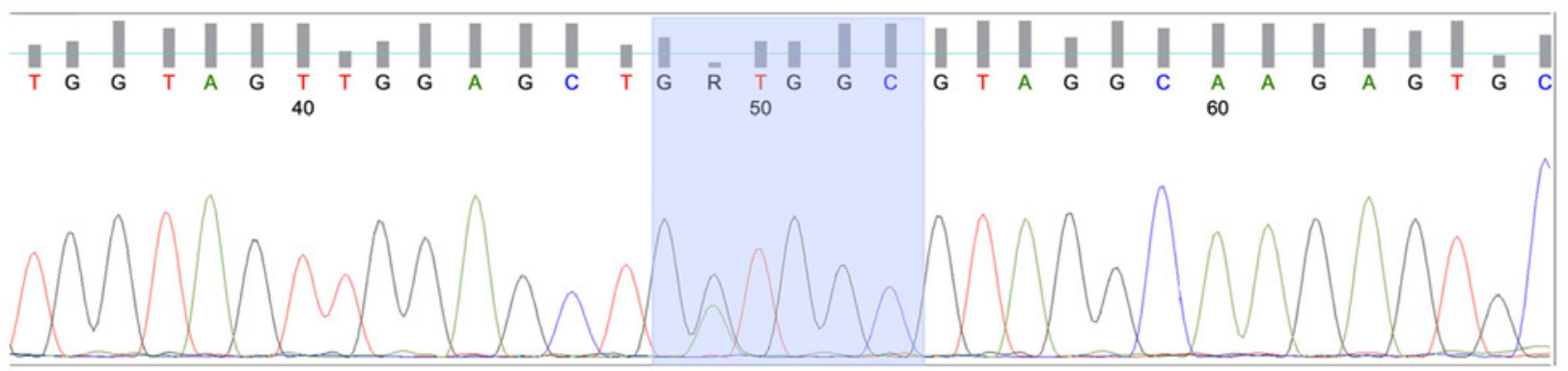

C

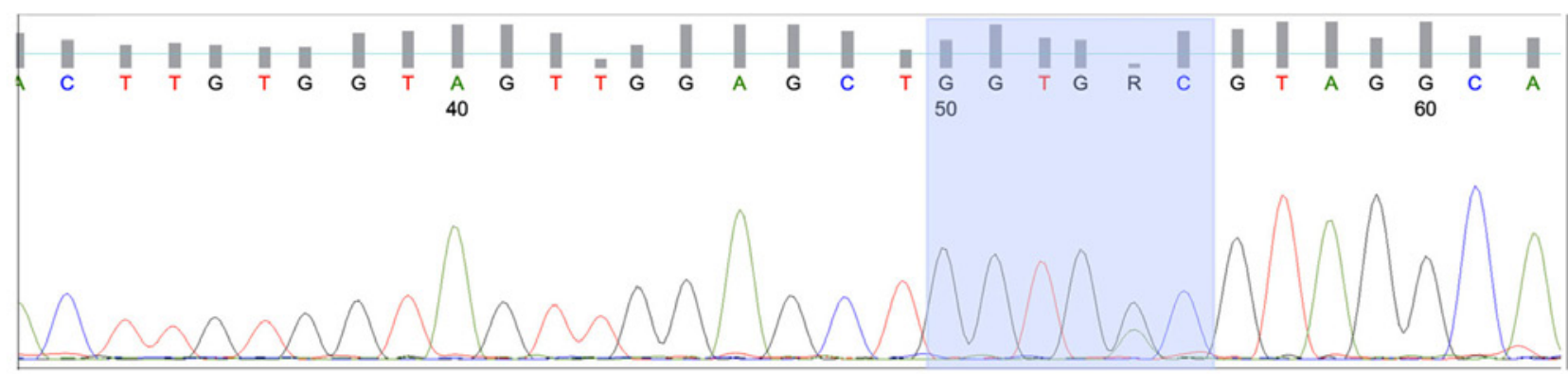

D

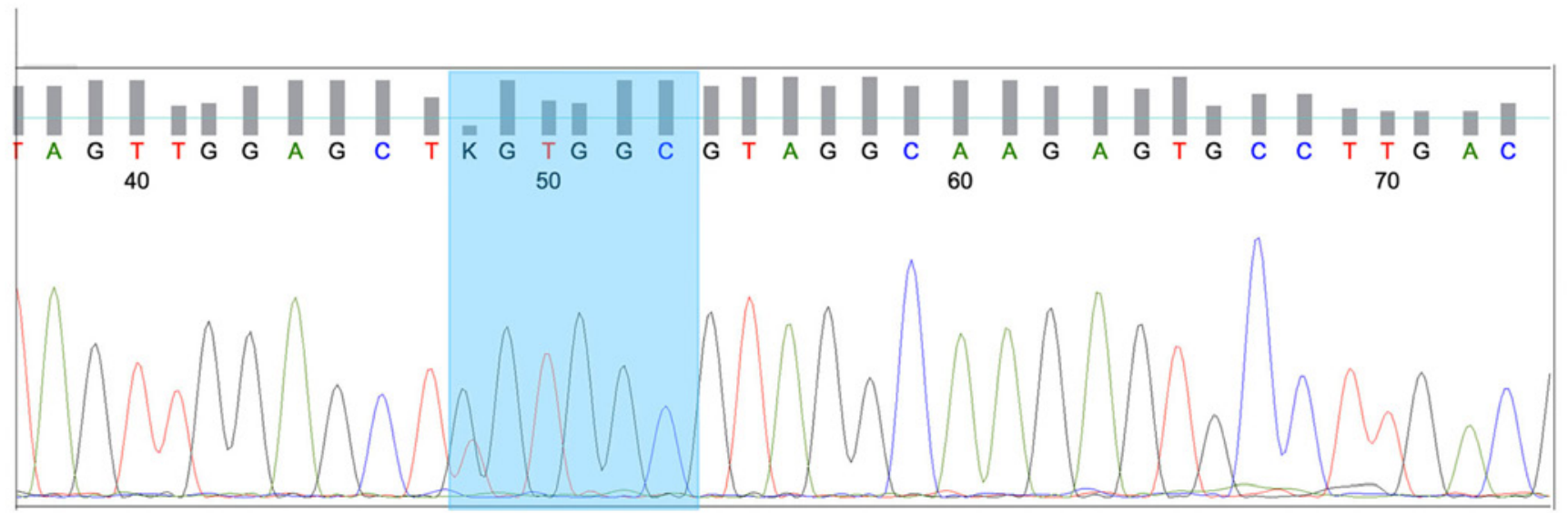

Figure 2. DNA isolated from patients was amplified by PCR using primers specific for codons $12 / 13$ of the KRAS gene. The PCR products were then subjected to Sanger sequencing and the results are presented as follows: (A) wild-type KRAS, (B) codon 12 GGT>GAT (GLY>ASP), (C) codon 13 GGC >GAC (GLY>ASP) and (D) codon 12 GGT>TGT (GLY>CYS).

Of the 13 tumors, $12(92.3 \%)$ had mutations in codon 12 , while 1 had a mutation in codon $13(7.7 \%)$. Of the 13 patients with CRC harboring KRAS mutations $6(46.1 \%)$ had a Gly12Asp mutation $4(30.8 \%)$ had a Gly12Val mutation, $2(15.4 \%)$ had a Gly12Cys mutation, and 1 (7.7\%) had a Gly13Asp mutation. Mutations from $\mathrm{G}$ to A occurred in 7/13 (53.8\%) of the patients, and from $\mathrm{G}$ to $\mathrm{T}$ in $6 / 13(46.2 \%)$ of the patients (Fig. 2). Mutations occurred at the first base of codon 12 or 13 in $2 / 13(15.4 \%)$ and at the second base in $11 / 13(84.6 \%)$ of the patients (Table II). 


\section{Discussion}

The present study investigated the association between clinicopathological factors and KRAS mutation status in Libyan patients with CRC and, except for the tumor site, no other significant associations were identified. This observation is consistent with the findings of a Saudi Arabian study (16) and the RASCAL multicenter study (17), which demonstrated a trend for higher mutation frequency in tumors of the left colon (51 and 58.8\%, respectively) compared to other sites $(16,17)$. This finding has been attributed to the increased exposure of the left-sided bowel lumen to ingested carcinogens and mutagens. By contrast, KRAS mutations have been reported to be slightly more frequent in the right colon compared with other sites (47 vs. 41\%, respectively) in studies from Saudi Arabia (18,19), while another study from Turkey reported that the KRAS mutation frequency was significantly higher in tumors located in the ascending colon (20).

There are several factors that may explain the variations in the observed frequencies of KRAS mutations according to tumor location in different studies. These include the use of different methodologies for the detection of mutations, with different ranges of sensitivity and specificity. Environmental factors have a major impact on different populations with diverse lifestyles, dietary habits, and variable exposures to carcinogens. Furthermore, the genetic polymorphisms in the carcinogen-metabolizing genes in different population groups must also be taken into consideration (21-24).

KRAS mutations were identified in $38.2 \%$ of all CRC patients in our study group. This finding was in agreement with previous reports that identified KRAS mutations in $30-40 \%$ of patients with CRC $(17,21)$. Consistent with previous reports, $92.3 \%$ of the studied tumors had a single base mutation at codon 12 of the KRAS gene, and $7.7 \%$ had a single base mutation at codon $13(17,21,22)$. The most common single base mutation $(46.1 \%)$ was a GGT to a GAT transition in codon 12 that resulted in a change from the amino acid glycine to aspartic acid (Gly12Asp). The frequency of KRAS mutations in our study group did not differ when compared with those of most other studies in Europe and the United States $(23,24)$. Previous studies that enrolled a relatively large number of Arabian patients with different stages of the disease, reported KRAS mutation rates of $28-56 \%$ in Saudi Arabia (19,25-27), 23-32\% in Tunisia $(22,28,29)$, $33-44 \%$ in Jordan $(30,31)$ and $42 \%$ in Egypt (32).

A number of factors may be associated with variations in the observed frequencies of KRAS mutations in different studies. Among these, environmental factors may be the most important parameter that may explain the difference in KRAS mutations between Libya and neighboring countries, such as Tunisia and Egypt, and western countries.

In conclusion, KRAS genetic alterations in codons 12 and 13 were found to be higher in this group of Libyan patients with CRC. However, further studies are required to fully elucidate the molecular background of CRC, by investigating other genes, such as APC, BRAF and NRAS, as well as other exons in the KRAS, HRAS and NRAS genes. Future studies of larger patient groups may provide more accurate information regarding the association between KRAS, HRAS and NRAS mutations and the clinicopathological characteristics of patients with CRC.

\section{Acknowledgements}

We would like to thank Dr Fatima Maetig and Dr Yachine Topov from NCI-Misurata for their pathological re-examinations of the CRC biopsies. We thank Dr Mohamed Elfagieh and the Scientific Committee of NCI-Misurata for their support for this project. We thank all the staff in the Parker Laboratory for their assistance during the project. We are grateful to Dr Alhusen Saad and Mr. Ahmed Hamouda from the Faculty of Engineering, Misurata University, for image processing of the sequencing.

\section{Funding}

MIP was supported by the SMART fellowship from the ICGEB, and research grants the South African Medical Research Council and the University of Cape Town.

\section{Availability of data and materials}

The datasets used and/or analyzed during the current study are available from the corresponding author on reasonable request.

\section{Authors' contributions}

AA conducted the experiments and the data analysis. MD assisted with the analysis of the obtained results. MA provided the clinical data of the patients with CRC. MIP was the co-supervisor of the study and critically revised the manuscript for important intellectual content. OA supervised the project, experimental design and data analysis, and edited the manuscript. MIP and OA have assessed and confirmed the authenticity of raw data. All the authors have read and approved the final version of the manuscript.

\section{Ethics approval and consent to participate}

All procedures performed in studies involving human participants were in accordance with the ethical standards of the National Cancer Institute-Misurata, the institutional research committee, and with the 1964 Helsinki Declaration and its later amendments or comparable ethical standards. All patients have signed the consent prior to the use of the studied tissues according to the regulations of the NCI-Misurata.

\section{Patient consent for publication}

Not applicable.

\section{Competing interests}

The authors declare that they have no competing interests.

\section{References}

1. Al-Sohaily S, Biankin A, Leong R, Kohonen-Corish M and Warusavitarne J: Molecular pathways in colorectal cancer. J Gastroenterol Hepatol 27: 1423-1431, 2012.

2. Ivanovich JL, Read TE, Ciske DJ, Kodner IJ and Whelan AJ: A practical approach to familial and hereditary colorectal cancer. Am J Med 107: 68-77, 1999.

3. Siegel RL, Miller KD and Jemal A: Cancer statistics, 2016. CA Cancer J Clin 66: 7-30, 2016. 
4. Fearon ER and Vogelstein B: A genetic model for colorectal tumourigenesis. Cell 61: 759-767, 1990

5. Lengauer C, Kinzler KW and Vogelstein B: Genetic instabilities in human cancers. Nature 396: 643-649, 1998.

6. Vogelstein B and Kinzler KW: Lessons from hereditary colorectal cancer. Cell 87: 159-170, 1996.

7. Arrington A, Heinrich E, Lee W, Duldulao M, Patel S, Sanchez J, Garcia-Aguilar J and Kim J: Prognostic and predictive roles of KRAS mutation in colorectal cancer. Int J Mol Sci 13 12153-12168, 2012.

8. Baines AT, Xu D and Der CJ: Inhibition of ras for cancer treatment: The search continues. Future Med Chem 3: 1787-1808, 2011.

9. Bamford S, Dawson E, Forbes S, Clements J, Pettett R, Dogan A, Flanagan A, Teague J, Futreal PA, Stratton MR and Wooster R The COSMIC (Catalogue of Somatic Mutations in Cancer) database and website. Br J Cancer 91: 355-358, 2004.

10. Tan C and Du X: KRAS mutation testing in metastatic colorectal cancer. World J Gastroenterol 18: 5171-5180, 2012.

11. Prior IA, Lewis PD and Mattos C: A comprehensive survey of ras mutations in cancer. Cancer Res 72: 2457-2467, 2012.

12. Jančík S, Drábek J, Radzioch D and Hajdúch M: Clinical relevance of KRAS in human cancers. J Biomed Biotechmol 2010: 150960, 2010.

13. Gideon P, John J, Frech M, Lautwein A, Clark R, Scheffler JE and Wittinghofer A: Mutational and kinetic analyses of the GTPase-activating protein (GAP)-p21 interaction: The C-terminal domain of GAP is not sufficient for full activity. Mol Cell Biol 12: 2050-2056, 1992.

14. Kinzler $\mathrm{K}$ and Vogelstein B: The genetic basis of cancer. In Cancer Gene Therapy. Curiel DT and Douglas JT (eds). Humana Press, Totowa, pp9-18, 2002.

15. Lee S, Brophy VH, Cao J, Velez M, Hoeppner C, Soviero S and Lawrence HJ: Analytical performance of a PCR assay for the detection of KRAS mutations (codons 12/13 and 61) in formalin-fixed paraffin-embedded tissue samples of colorectal carcinoma. Virchows Arch 460: 141-149, 2012

16. Bader T and Ismail A: Higher prevalence of KRAS mutations in colorectal cancer in Saudi Arabia: Propensity for lung metastasis. Alexandria J Med 50: 203-209, 2014.

17. Andreyev HJ, Norman AR, Clarke PA, Cunningham D and Oates J: Kirsten ras mutations in patients with colorectal cancer: The multicenter RASCAL study. J Nat Cancer Inst 90: 675-684, 1998.

18. Zekri J, Rizvi A, Al-Maghrabi J and Bin Sadiq B: K-Ras in colorectal cancer tumors from Saudi patients: Frequency, clinicopathological association and clinical outcome. Open Colorectal Cancer J 5: 22-27, 2012

19. Abubaker J, Bavi P, Al-Haqawi W, Sultana M, Al-Harbi S, Al-Sanea N, Abduljabbar A, Ashari LH, Alhomoud S, Al-Daye F, et al: Prognostic significance of alterations in KRAS isoforms KRAS-4A/4B and KRAS mutations in colorectal carcinoma. J Pathol 219: 435-445, 2009.

20. Gorukmez O, Yakut T, Gorukmez O, Sag SO, Karkucak M and Kanat O: Distribution of KRAS and BRAF mutations in metastatic colorectal cancers in Turkish patients. Asian Pac J Cancer Prev 17: 1175-1179, 2016
21. Smith G, Carey FA, Beattie J, Wilkie MJ, Lightfoot TJ, Coxhead J, Garner RC, Steele RJ and Wolf C: Mutations in APC, Kirsten-ras, and p53-alternative genetic pathways to colorectal cancer. Proc Natl Acad Sci 99: 9433-9438, 2002.

22. Aissi S, Buisine M, Zerimech F, Kourda N, Moussa A, Manai M and Porchet N: KRAS mutations in colorectal cancer from Tunisia: Relationships with clinicopathological variables and data on TP53 mutations and microsatellite instability. Mol Biol Rep 40: 6107-6112, 2013.

23. Edkins S, O'Meara S, Parker A, Stevens C, Reis M, Jones S, Greenman C, Davies H, Dalgliesh G, Forbes S, Hunter C, et al: Recurrent KRAS codon 146 mutations in human colorectal cancer. Cancer Biol Ther 5: 928-932, 2006.

24. Licar A, Cerkovnik P, Ocvirk J and Novakovic S: KRAS mutations in Slovene patients with colorectal cancer: Frequency, distribution and correlation with the response to treatment. Int J Oncol 36: 1137-1144, 2010.

25. Zekri J, Al-Shehri A, Mahrous M, Al-Rehaily S, Darwish T, Bassi S, El Taani H, Al Zahrani A, Elsamany S, Al-Maghrabi J and Sadiq BB: Mutations in codons 12 and 13 of K-ras exon 2 in colorectal tumors of Saudi Arabian patients: Frequency, clinicopathological associations, and clinical outcomes. Genet Mol Res 16: 4238, 2017.

26. Beg S, Siraj A, Prabhakaran S, Bu R, Al-Rasheed M, Sultana M, Qadri Z, Al-Assiri M, Sairafi R, Al-Sanea N, et al: Molecular markers and pathway analysis of colorectal carcinoma in the Middle East. Cancer 121: 3799-3808, 2015.

27. Dallol A, Buhmeida A, Al-Ahwal MS, Al-Maghrabi J, Bajouh O, Al-Khayyat S, Alam R, Abusanad A, Turki R, Elaimi A, et al: Clinical significance of frequent somatic mutations detected by high-throughput targeted sequencing in archived colorectal cancer samples. J Transl Med 14: 118, 2016.

28. Sammoud S, Khiari M, Semeh A, Amine L, Ines C, Amira A, Lilia K, Taher K, Sabeh M and Saadia B: Relationship between expression of ras p21 oncoprotein and mutation status of the K-ras gene in sporadic colorectal cancer patients in Tunisia. Applied Immunohistochem Mol Morphol 20: 146-152, 2012.

29. Aissi S, Buisine M, Zerimech F, Kourda N, Moussa A, Manai M and Porchet N: Somatic molecular changes and histopathological features of colorectal cancer in Tunisia. World J Gastroenterol 19: 5286-5294, 2013

30. Elbjeirami W and Sughayer M: KRAS mutations and subtyping in colorectal cancer in Jordanian patients. Oncol Lett 4: 705-710, 2012.

31. Gumus M, Dane F, Karabulut B, Uygun K, Orhan B, Aydin K and Ozen R: Interim results of observational study to determine $\mathrm{K}$-ras mutation rates in patients with metastatic colorectal cancer in Turkey. Eur J Cancer 49 (Suppl): S571-S571, 2013.

32. Elsabah $\mathrm{M}$ and Adel I: Immunohistochemical assay for detection of K-ras protein expression in metastatic colorectal cancer. J Egypt Nat Cancer Inst 25: 51-56, 2013.

This work is licensed under a Creative Commons Attribution-NonCommercial-NoDerivatives 4.0 International (CC BY-NC-ND 4.0) License. 